\title{
Evolutionary models for low-mass stars and brown dwarfs: Uncertainties and limits at very young ages
}

\author{
I. Baraffe $e^{1,2}$, G. Chabrier ${ }^{1}$, F. Allard ${ }^{1}$, and P. H. Hauschildt ${ }^{3}$ \\ 1 C.R.A.L (UMR 5574 CNRS), École Normale Supérieure, 69364 Lyon Cedex 07, France \\ e-mail: chabrier, fallard@ens-lyon.fr \\ 2 Max-Planck-Institut für Astrophysik, Karl-Schwarzschildstr.1, 85748 Garching, Germany \\ 3 Center for Simulational Physics, University of Georgia Athens, GA 30602-2451, USA \\ e-mail: yeti@hobbes.physast.uga.edu \\ Received 25 September 2001 / Accepted 15 November 2001
}

\begin{abstract}
We analyse pre-Main Sequence evolutionary tracks for low mass stars with masses $m \leq 1.4 M_{\odot}$ based on the Baraffe et al. (1998) input physics. We also extend the recent Chabrier et al. (2000) evolutionary models based on dusty atmosphere to young brown dwarfs down to one mass of Jupiter. We analyse current theoretical uncertainties due to molecular line lists, convection and initial conditions. Simple tests on initial conditions show the high uncertainties of models at ages $\lesssim 1$ Myr. We find a significant sensitivity of atmosphere profiles to the treatment of convection at low gravity and $T_{\text {eff }}<4000 \mathrm{~K}$, whereas it vanishes as gravity increases. This effect adds another source of uncertainty on evolutionary tracks at very early phases. We show that at low surface gravity $(\log g \lesssim 3.5)$ the common picture of vertical Hayashi lines with constant $T_{\text {eff }}$ is oversimplified. The effect of a variation of initial deuterium abundance is studied. We compare our models with evolutionary tracks available in the literature and discuss the main differences. We finally analyse to what extent current observations of young systems provide a good test for pre-Main Sequence tracks.
\end{abstract}

Key words. stars: low-mass, brown dwarfs - stars: evolution - stars: pre-main sequence

\section{Introduction}

The development of a new generation of stellar evolution models based on the accurate coupling between interior and atmosphere models yielded a major advance in the description of very-low-mass stars (VLMS; Baraffe et al. 1995, 1997, 1998, BCAH98) and substellar objects (brown dwarfs BD and giant planets GP; Burrows et al. 1997 for objects with $T_{\text {eff }}<2000 \mathrm{~K}$; Chabrier et al. 2000a, CBAH00). One of the main advantages of such models over previous generation models is the direct comparison between theory and observation in colour-colour and colour-magnitude diagrams (CMD). Several observational tests now assess the validity of the theory devoted to the description of low-mass $\left(\lesssim 1 M_{\odot}\right)$ astrophysical objects (see Allard et al. 1997; Chabrier \& Baraffe 2000, for recent reviews). Although some discrepancies between models and observations still remain, uncertainties due to the input physics are now significantly reduced.

In previous papers, we have developed evolutionary models of VLMS and BDs (BCAH98; CBAH00), taking into account the most recent improvements of the physics describing the interior (equation of state for dense plasmas, screening factors) and the atmosphere

Send offprint requests to: I. Baraffe, e-mail: ibaraffe@ens-lyon.fr (molecular opacities, formation of dust) devoted to the analysis of objects with an age $t \gtrsim 100 \mathrm{Myr}$. Comparison between observations and models for very young objects $(t<100 \mathrm{Myr})$ are more uncertain, in particular from the observational viewpoint, mainly for two reasons: (1) extinction due to the surrounding dust modifies both the intrinsic magnitude and the colours of the objects and (2) spectra of very young objects ( $t \lesssim 1 \mathrm{Myr}$ ) may still be affected by the presence of an accretion disk or circumstellar material residual from the protostellar stage. On the theoretical side, an important source of uncertainty at the very early stages of evolution is the choice of the initial conditions, resulting from prior protostellar collapse and accretion phases. The simple picture of non-accreting objects contracting from large initial radii, as used in our models, is clearly an idealized description of reality (Stahler et al. 1980; Stahler 1983, 1988; Hartmann et al. 1997 and references therein).

In spite of these uncertainties, numerous surveys are devoted to very young clusters because of the potential detection of substellar objects down to planetary masses (Zapatero et al. 2000; Lucas et al. 2001), and the determination of the very (sub)stellar initial mass functions (IMF), free of dynamical evolution effects that affect older clusters. Consequently, a wealth of data for low mass objects with ages spanning $\sim 1-10 \mathrm{Myr}$ is available and 
provides the basis for a better understanding of the early phases of stellar evolution. Given the reliability of the present theory for VLMS and $\mathrm{BD}$, a confrontation of the models with the observational data of such very young objects provides important insight into the early epoch of protostellar collapse and accretion phases. For this purpose, however, a correct appreciation of the model uncertainties and limitations is required.

For this reason, this paper is specifically devoted to the analysis of evolutionary models by BCAH98 and CBAH00 at early phases of evolution $(t \lesssim 100 \mathrm{Myr})$. The input physics is briefly recalled in Sect. 2 , followed by a discussion of uncertainties specific to evolutionary tracks at early ages (Sect. 3). Our models are compared to other evolutionary tracks available in the literature in Sect. 4 and the confrontation to observations is presented in Sect. 5 .

\section{Model description}

\subsection{Evolutionary tracks}

The models analysed in the present paper are based on the input physics already described in BCAH98 and CBAH00. Both sets of models use the same ingredients describing the stellar interior but use different sets of atmosphere models, which provide the outer boundary conditions and the synthetic spectra. The BCAH98 evolutionary tracks are based on the non-grey atmosphere models by Hauschildt et al. (1999a). These models are dustfree and are appropriate to the description of objects with effective temperatures $T_{\text {eff }} \gtrsim 2300 \mathrm{~K}$. The CBAH00 models are based on atmospheres including the formation and opacity of dust (Allard et al. 2001, hereafter DUSTY models). As illustrated in CBAH00, dust must be taken into account in order to explain the near-IR colors of late Mdwarfs and L-dwarfs. The latter models are thus more appropriate to the description of objects with $T_{\text {eff }} \gtrsim 2300 \mathrm{~K}$. As emphasized in CBAH00, the DUSTY models are not appropriate for the description of spectral and photometric properties of methane dwarfs $\left(T_{\text {eff }}<1600 \mathrm{~K}\right)$, which require a different treatment of dust (the so-called COND models in CBAH00 and Allard et al. 2001). Evolutionary models for such cool objects will be presented elsewhere (Chabrier et al. 2001, in preparation).

The BCAH98 grid covered a mass range from $0.02 M_{\odot}$ to $1.4 M_{\odot}$, for ages $\geq 1 \mathrm{Myr}$ up to the Main Sequence for stars. Originally, the CBAH00 grid covered masses from $0.01 M_{\odot}$ to $0.1 M_{\odot}$ for ages $\geq 10$ Myr. In the present work, we extend it down to $1 M_{\mathrm{J}}\left(10^{-3} M_{\odot}\right)$ and ages $\geq 1 \mathrm{Myr}$. Figure 1 presents the complete grid of models in a Hertzsprung-Russell diagram (HRD) from $0.001 M_{\odot}$ to $1.4 M_{\odot}$. The small variation of radius with mass and age in the substellar regime (see e.g. Chabrier \& Baraffe 2000) yields the merging towards very similar tracks below the hydrogen-burning limit. Figure 2 displays the time evolution of the effective temperature and luminosity for selected masses. Objects below $2 M_{\mathrm{J}}$ evolve essentially with $T_{\text {eff }}<1600 \mathrm{~K}$ (see Figs. 1 and 2), even at very early ages.

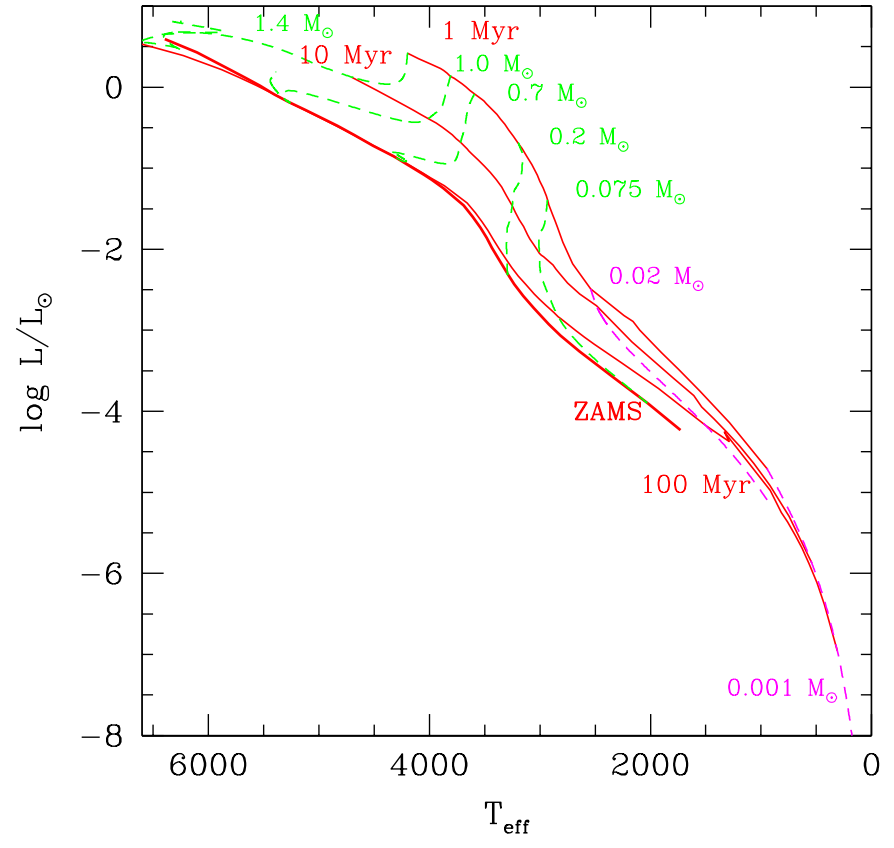

Fig. 1. Evolutionary tracks in the Hertzsprung-Russell diagram for masses from $1.4 M_{\odot}$ to $0.001 M_{\odot}$ (dashed lines) and ages spanning from $1 \mathrm{Myr}$ to the ZAMS (for stars). Several isochrones for 1, 10 and $100 \mathrm{Myr}$ are indicated by solid lines from right to left. The location of the ZAMS for stars down to $0.075 M_{\odot}$ is also indicated (left solid line).

Their atmospheric properties are thus better described by the COND models.

The initial conditions of the models are described in the next section. The stellar/substellar transition is located at $m \sim 0.075 M_{\odot}$. Below this limit, objects become partially degenerate and their nuclear energy production cannot compensate the energy lost by radiation (e.g. $\left.L_{\text {nuc }}<L\right)$, which is required to reach the Main Sequence. The deuterium burning minimum mass is $m \sim 0.012 M_{\odot}$ (Saumon et al. 1996; Chabrier et al. 2000b). The initial D-burning phase lasts less than $1 \mathrm{Myr}$ for $m \gtrsim 0.2 M_{\odot}$, between 1 and $5 \mathrm{Myr}$ for $0.05 \lesssim m \lesssim 0.2 M_{\odot}$ and almost $20 \mathrm{Myr}$ for a $0.02 M_{\odot}$ brown dwarf (see Chabrier et al. 2000b, for details).

As shown in CBAH00, grains have little effects on the evolution of $L(t)$ and $T_{\text {eff }}(t)$, because of the reduced dependence of evolution upon opacity, $L(t) \propto \kappa_{\mathrm{R}}^{\sim 1 / 3}$, $T_{\text {eff }}(t) \propto \kappa_{\mathrm{R}}^{\sim 1 / 10}$ (Burrows \& Liebert 1993). We verified that the difference between the different $\mathrm{TiO}$ and $\mathrm{H}_{2} \mathrm{O}$ molecular line lists used in BCAH98 and CBAH00 models (see Sect. 3 in CBAH00), respectively, affect essentially the outer atmospheric layers, and thus the synthetic spectra and colors, but not the deeper atmospheric layers, and thus the outer boundary conditions. The effect of these different molecular linelists on the evolution of the effective temperature $T_{\text {eff }}(t)$ and the bolometric luminosity $L(t)$ is small, less than $100 \mathrm{~K}$ in $T_{\text {eff }}$ and $10 \%$ in $L$ at a given age. This is illustrated in Fig. 2, where tracks based on the BCAH98 input physics (solid lines) are compared to the 


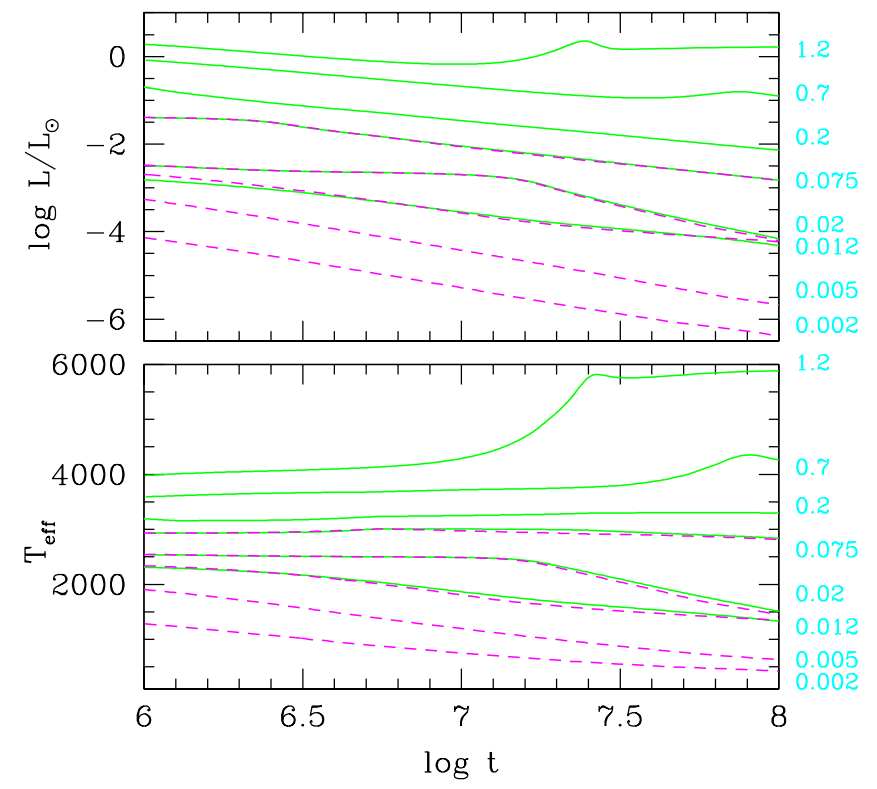

Fig. 2. Evolution of luminosity and effective temperature as a function of time (in yr) for masses from $1.2 M_{\odot}$ to $0.002 M_{\odot}$. Masses (in $M_{\odot}$ ) are indicated on the right hand side of the figure. The solid lines are the BCAH98 models. The dashed lines are dusty-models based on the CBAHOO input physics.

CBAH00 tracks (dashed lines) for the same masses (0.075, 0.02 and $\left.0.012 M_{\odot}\right)$. As stressed in CBAH00 and Baraffe et al. (2001), the computation of more reliable $\mathrm{H}_{2} \mathrm{O}$ and, to a lesser extend, $\mathrm{TiO}$ linelists is badly needed to solve this shortcoming in the present theory.

\section{Main uncertainties: Initial model and convection}

\subsection{Initial conditions}

As mentioned in the previous section, although shortcomings still remain in current molecular opacities, the resulting uncertainty on the evolution is small. One of the main source of uncertainty for models at early stages of evolution is the choice of the initial conditions. Most of low mass pre-Main Sequence (PMS) models available in the literature (D'Antona \& Mazzitelli 1994, 1997; Burrows et al. 1997; BCAH98; Siess et al. 2000) start from arbitrary initial conditions, totally independent on the outcome of the prior proto-stellar collapse and accretion phases. The initial configuration is that of a fully convective object starting its contraction along the Hayashi line from arbitrary large radii. Evolution starts prior to or at central deuterium ignition, with initial central temperature $\sim 5 \times 10^{5} \mathrm{~K}$. According to studies of low-mass protostellar collapse and accretion phases, such initial conditions are oversimplified, and low mass objects should rather form with relatively small radii (Hartmann et al. 1997, and references therein). Based on spherical accretion protostellar models, Stahler $(1983,1988)$ defined a birthline in the Hertzsprung-Russell diagram where young objects become visible. Evolutionary tracks should then start from this birthline, which fixes the age $t=0$. Ages determined from models based on the above-mentioned oversimplified conditions should then be corrected accordingly, with substantial corrections for systems younger than a few Myr (see Palla \& Stahler 1999). Furthermore, collapse and accretion are unlikely to proceed spherically. Spherical collapse does not consider angular momentum transport, an important issue of the early phases, which affects the subsequent cooling and formation of the protostar. Hartmann et al. (1997) recently illustrate the sensitivity of the birthline locus assuming that accretion proceeds through a disk rather than spherically. This work stresses again the high uncertainty of assigning ages from HRD positions for the youngest objects.

This analysis demonstrates convincingly that assigning an age to objects younger than a few Myr is totally meaningless when the age is based on models using oversimplified initial conditions. As shown in the next section, for $t \lesssim 1 \mathrm{Myr}$, the evolutionary tracks themselves are sensitive to the initial conditions, whereas after a few Myr, the models converge toward the same track. This is the main reason why we provide confidently evolutionary models for ages $t \geq 1 \mathrm{Myr}$, considering that below such ages, models are too uncertain. To solve this substantial uncertainty requires the consistent evolution between the $3 \mathrm{D}$ collapse of the protostellar phase and the subsequent PMS evolution.

\subsubsection{Effect of the initial radius and the mixing length parameter}

In this section we examine the effect of the variation of the initial radius (or initial gravity) in the initial conditions. For this purpose we have extended our grid of atmosphere models to gravities $\log g<3.5$. We have checked the validity of the plane-parallel approximation used in these models by comparison with similar atmosphere models which take into account the effects of spherical geometry. These effects are found to become important only for surface gravities $\log g \lesssim 2$ (Hauschildt et al. 1999b; Allard et al. 2000), well below the range of gravities characteristic of the evolution of young low mass objects.

We first calculate a set of models, labeled (A), with initial radii fixed to obtain initial surface gravities $\log g \sim$ 3-3.5 and initial thermal time-scales $t_{\text {th }} \sim$ a few Myr. For masses above the deuterium burning minimum mass (DBMM), $m \gtrsim 0.02 M_{\odot}$, such tracks start at deuterium ignition, with central temperature $\sim 5 \times 10^{5}-10^{6} \mathrm{~K}$, defining a Zero-Age-Deuterium Burning Sequence. Such initial conditions are similar to that used in BCAH98 and CBAH00. A second set of models, labeled (B), starts with larger radii such that the initial surface gravity $\log g \sim$ 2.5. These initial models are more luminous than the previous ones, with central temperatures below $5 \times 10^{5} \mathrm{~K}$ and initial thermal time-scales $t_{\mathrm{th}} \sim 10^{5} \mathrm{yr}$.

We also analyse the sensitivity of the models to the mixing length $l_{\text {mix }}$, characteristic of the mixing length 

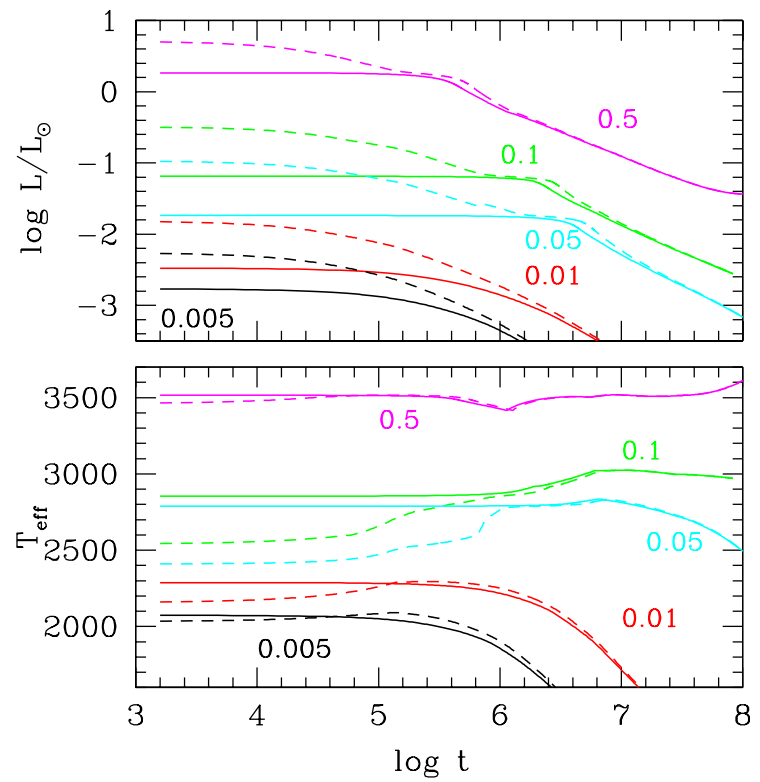

Fig. 3. Effect of the initial radius on the evolution of luminosity and effective temperature as a function of time (in yr) for several masses (indicated near the curves in $M_{\odot}$ ). The solid lines correspond to the first set of models (A) with initial gravity $\log g \sim 3-3.5$ and the dashed lines correspond to models (B) starting with initial gravity $\log g \sim 2.5$. The mixing length is $l_{\text {mix }}=H_{\mathrm{P}}$.

formalism (MLT) used to describe convection. We used two different values of the mixing length parameter $\alpha_{\text {mix }}{ }^{1}=1$ and 2 (see Sect. 3.2 for justifications).

The time evolution of $L$ and $T_{\text {eff }}$ for models (A) (solid lines) and (B) (dashed lines) is displayed in Fig. 3 for several masses and for $\alpha_{\text {mix }}=1$. Figure 3 shows the importance of the initial radius on $T_{\text {eff }}$, and thus on $L$, during the first Myr of evolution. After a few Myr, however, the tracks based on different initial radii merge, for a given mass. For masses ranging from $\sim 0.01$ to $0.5 M_{\odot}$, the two sets of models start at very different $T_{\text {eff }}$. Models starting with the lowest gravity are cooler by up to several hundreds K compared to initially denser, less luminous models with the same mass. Note also that for models (B), $T_{\text {eff }}$ increases during the early evolution, under contraction, in contrast to the first set of models with initial $\log g \gtrsim 3.0$. This is the consequence of the different surface gravities, which strongly affect the atmosphere profiles for $T_{\text {eff }}=$ 2200-3500 K, as illustrated in Fig. 4. As shown in this figure, for this range of effective temperatures, the atmosphere profiles also show an extreme sensitivity to the mixing length $l_{\text {mix }}$ at low gravity $(\log g<3.5)$ but become rather insensitive to a variation of this parameter for $\log g \geq 3.5$ (see Fig. 4b).

Such a sensitivity of the atmospheric profiles to gravity and mixing length in this range of $T_{\text {eff }}$ stems from the less efficient formation of molecular $\mathrm{H}_{2}$ as gravity decreases. Figure 5 displays the fraction of $\mathrm{H}_{2}$ along the atmosphere profiles shown in Fig. 4 (below the photosphere).

\footnotetext{
${ }^{1} \alpha_{\text {mix }}=l_{\text {mix }} / H_{\mathrm{P}}$, with $H_{\mathrm{P}}$ the pressure scale-height.
}

For $T_{\text {eff }}=2500 \mathrm{~K}$ and $\log g=2.5$, the fraction $n_{\mathrm{H}_{2}}$ of $\mathrm{H}_{2}$ decreases rapidly along the inner profile. For $\alpha_{\text {mix }}=1$, it becomes negligible at optical depth $\tau=100$, where the boundary condition for the inner structure is defined (see Chabrier \& Baraffe 1997, their Sect. 2.5). For $\log g \geq 3.0$ (see Fig. 5), $n_{\mathrm{H}_{2}}$ exceeds $20 \%$, even in the deepest atmospheric layers.

As described in Chabrier \& Baraffe (2000, Sect. 2.2.2), hydrogen atom recombination yields a rapid increase of the $\mathrm{H}_{2}$ Collision Induced Absorption opacity $\left(\kappa_{\text {CIA }} \propto\right.$ $n_{\mathrm{H}_{2}}^{2}$ ) and a decrease of the adiabatic gradient. Both effects favor convection, yielding a flatter atmosphere profile, i.e. a decrease of $T$ at fixed $P$. This in turn favors the formation of $\mathrm{H}_{2}$ and increases $n_{\mathrm{H}_{2}}$ at fixed $P$, increasing again the opacity and the efficiency of convection. This illustrates the non-linear response of the atmospheric structure to the formation of $\mathrm{H}_{2}$, as soon as its fraction becomes significant. It explains the huge effect of gravity when this latter increases from $\log g=2.5$ to 3.0 on the atmospheric profile, as illustrated in Fig. 4a. The decrease of $\nabla_{\text {ad }}$ in regions of $\mathrm{H}_{2}$ formation favors adiabatic convection, limiting the extension of super-adiabatic layers and thus the sensitivity of the thermal profile to the mixing length parameter. This explains the strong dependence of the atmosphere profile on $\alpha_{\text {mix }}$ for $\log g=2.5$ (see Fig. 4b). At higher gravities convection described by the MLT is essentially adiabatic and almost insensitive to the choice of $\alpha_{\operatorname{mix}}$ (see Sect. 3.2).

A similar behavior is found in the range of effective temperatures $2200 \mathrm{~K} \lesssim T_{\text {eff }} \lesssim 4000 \mathrm{~K}$. Above $\sim 4000 \mathrm{~K}$, the structure is too hot for $\mathrm{H}_{2}$ to form in significant fraction whereas below $T_{\text {eff }} \sim 2200 \mathrm{~K}$, the outer layers are dense and cool enough for $\mathrm{H}_{2}$ to form efficiently, even at $\log g=2.5$.

The drastic modification of the atmospheric profile, for $\alpha_{\text {mix }}=1$, as gravity increases from $\log g=2.5$ to $\log g \geq$ 3.0 explains the different $T_{\text {eff's }}$ for models starting from different initial radii (Fig. 3). For models (B), starting with $\log g=2.5$, gravity increases as contraction proceeds and favors $\mathrm{H}_{2}$ molecular formation, yielding significantly flatter atmospheric profiles, as mentioned above. This yields a significant increase of $T_{\text {eff }}$ at fixed mass $m$ as contraction proceeds (Chabrier \& Baraffe 2000, Sect. 3.2). For $\alpha_{\text {mix }}=$ 2 , the sensitivity of atmosphere profiles to $g$ is less pronounced, but still yields up to $200 \mathrm{~K}$ differences between models (A) and (B) at a given age.

The effect on evolutionary tracks in a HRD is displayed in Fig. 6. Note that evolution along the Hayashi line does not necessarily proceed at constant $T_{\text {eff }}$, because of the effects described above. The common picture of vertical (constant $T_{\text {eff }}$ ) Hayashi tracks is therefore an oversimplified picture of PMS evolution. As demonstrated in the pioneering papers of Hayashi (1961) and Hayashi \& Nakano (1963), fully convective and adiabatic objects which have $\mathrm{H}_{2}$ dissociation zones contract almost vertically in the HRD. The very low value of $\nabla_{\text {ad }}(<0.1)$ in such zones is responsible for such evolution at constant $T_{\text {eff }}$. Taking into account super-adiabaticity in convective layers displace 

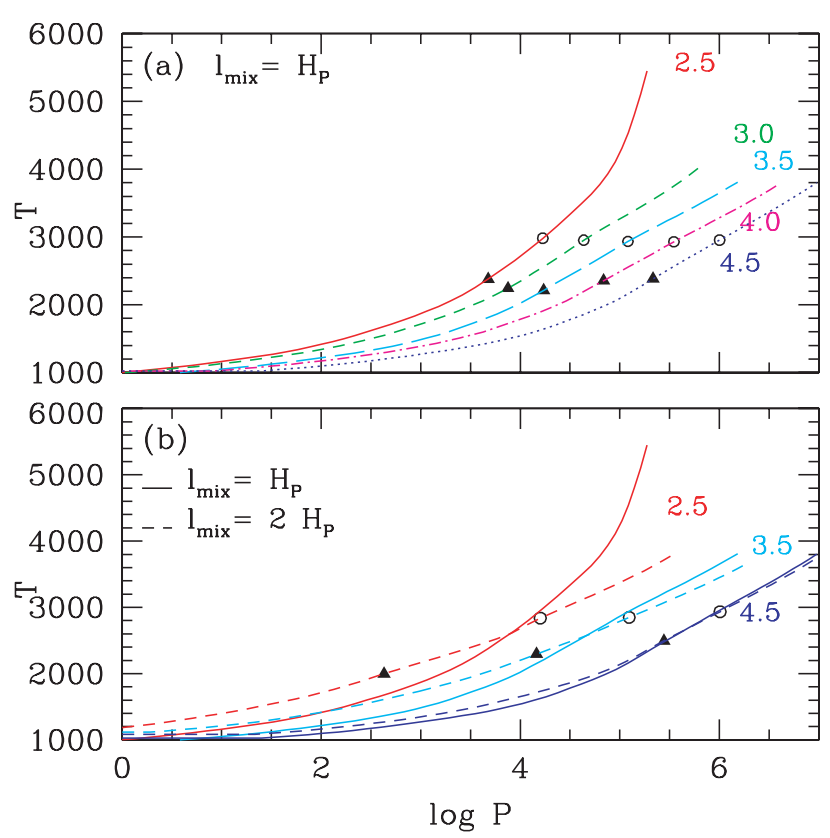

Fig. 4. a) Gravity effect on $P-T$ atmosphere profiles with $T_{\text {eff }}=2500 \mathrm{~K}$ and $l_{\text {mix }}=H_{\mathrm{P}}$. Pressure is in dyne $\mathrm{cm}^{-2}$ and temperature in $\mathrm{K}$. The surface gravity varies from $\log g=2.5$ to 4.5 , as indicated on the figure. The open circles on each curve correspond to an optical depth $\tau=1$. The filled triangles indicate the onset of convection. The inner atmosphere profiles stop at $\tau=100$, where the outer boundary condition for the inner structure is defined. b) Effect of a variation of $l_{\text {mix }}$ for atmosphere profiles with $T_{\text {eff }}=2500 \mathrm{~K}$ and gravity $\log g=$ $2.5,3.5$ and 4.5. Symbols on the dashed curve are the same as in a).

the Hayashi line toward lower effective temperature. This is equivalent to a decrease of $\alpha_{\text {mix }}$ in our calculations. Hayashi \& Nakano (1963) have also shown that omitting the presence of $\mathrm{H}_{2}$ molecules yields an evolution proceeding at decreasing $T_{\text {eff }}$ (from the left to the right in a HRD), rather than at constant $T_{\text {eff }}$. Although based on a simplified treatment of the atmospheric properties, opacities and equation of state, these basic works already illustrated the extreme sensitivity of the shape of PMS tracks for low mass objects to super-adiabaticity and to the degree of $\mathrm{H}_{2}$ formation/dissociation.

Models (A) and (B) follow the same track for a given mass and $\alpha_{\text {mix }}$, but do not reach the same position at the same age. Significant differences appear at ages $\lesssim 1 \mathrm{Myr}$ but vanish after a few Myr. We thus consider 1 Myr as the characteristic time required to forget our arbitrary initial conditions and below which models are too sensitive to input physics and thus too uncertain.

\subsubsection{Effect of the initial deuterium abundance}

Deuterium burning plays a key role during the protostellar collapse phase (Stahler 1988) and the following $~ 10 \mathrm{Myr}$ of evolution. In a previous paper, we focused on the initial deuterium burning phase (Chabrier et al. 2000b), adopting an initial mass fraction $\left[{ }^{2} D_{0}\right]=2 \times 10^{-5}$, characteristic
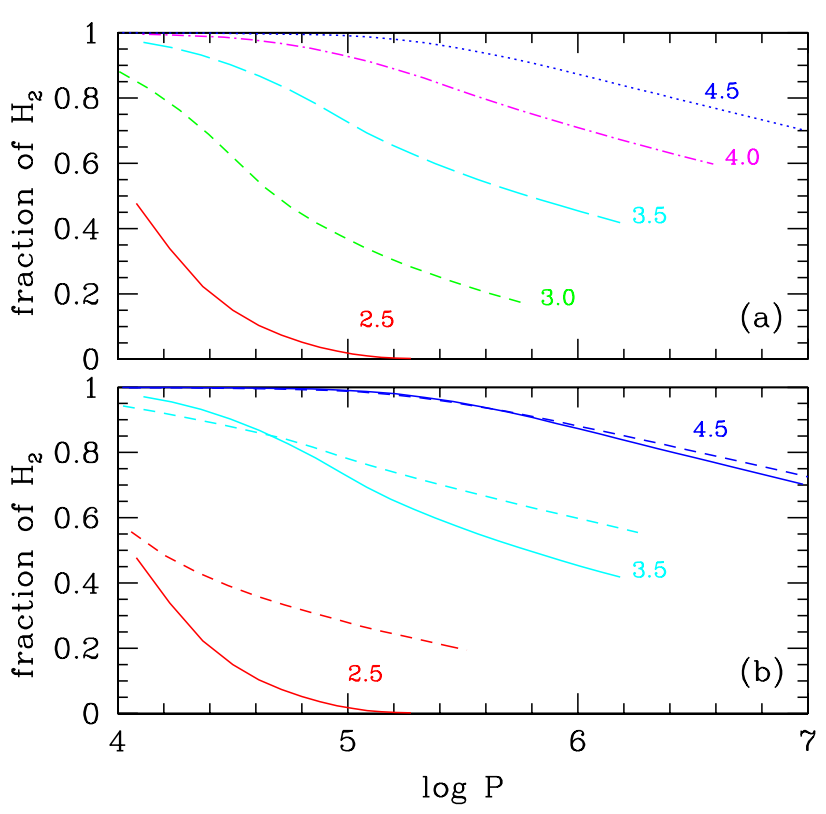

Fig. 5. a) Number fraction of molecular $\mathrm{H}_{2}$ as a function of $P$ (in dyne $\mathrm{cm}^{-2}$ ) along the profiles displayed in Fig. 4 with $T_{\text {eff }}=$ $2500 \mathrm{~K}, l_{\text {mix }}=H_{\mathrm{P}}$ and different gravities $\log g$ (indicated near the curves). b) Same as in a) for $l_{\text {mix }}=H_{\mathrm{P}}$ (solid lines) and $l_{\text {mix }}=2 H_{\mathrm{P}}$ (dashed lines).

of the local interstellar medium (hereafter LISM, Linsky 1998). In the present section we examine the effect of a variation of $\left[{ }^{2} D_{0}\right]$ by a factor of 2 on the early stages of evolution. Such a variation is motivated by a recent deuterium abundance determination towards quasars, which suggests a primordial abundance only slightly larger (by less than a factor of 2) than the LISM value (O'Meara et al. 2001).

The main effect of a variation of the initial $\left[{ }^{2} D_{0}\right]$ is the modification of the age at a given $L$ and $T_{\text {eff }}$, with the most important effect for masses $m \lesssim 0.07 M_{\odot}$ (Fig. 7). For a standard $\left[{ }^{2} D_{0}\right]$, a $0.07 M_{\odot}$ depletes by a factor of $100 \mathrm{its}$ initial deuterium content within $\sim 3 \mathrm{Myr}$ and a $0.02 M_{\odot}$ needs $\sim 17 \mathrm{Myr}$ (see Chabrier et al. 2000b). If $\left[{ }^{2} D_{0}\right]$ is increased by a factor of 2 , the $0.07 M_{\odot}$ brown dwarf then needs $\sim 5 \mathrm{Myr}$ and the $0.02 M_{\odot}$ requires $\sim 26 \mathrm{Myr}$ for the same depletion factor. A $0.015 M_{\odot}$ brown dwarf requires $50 \mathrm{Myr}$ in the standard case and $70 \mathrm{Myr}$ if $\left[{ }^{2} D_{0}\right]$ is twice as large to reach the $99 \%$ depletion limit. Conversely, if $\left[{ }^{2} D_{0}\right]$ is smaller by a factor of 2 , the $99 \%$ depletion limit is reached in 1.7, 12 and $40 \mathrm{Myr}$ for respectively $0.07,0.02$ and $0.015 M_{\odot}$. If the initial $\left[{ }^{2} D_{0}\right]$ is as small as $2 \times 10^{-6}$, e.g. a factor of 10 smaller than the standard LISM value, all objects with masses $m \gtrsim 0.04 M_{\odot}$ depletes their initial deuterium within 2 Myr.

The effect of a variation of $\left[{ }^{2} D_{0}\right]$ on isochrones in CMDs, however, remains small, compared to other sources of uncertainties at young ages (extinction, $T_{\text {eff }}$ calibration, initial models) and can be ignored for this present. If the improvement of observable techniques in the future, however, allows the detection of deuterium in the atmosphere of young objects (Chabrier et al. 2000b), this effect needs 


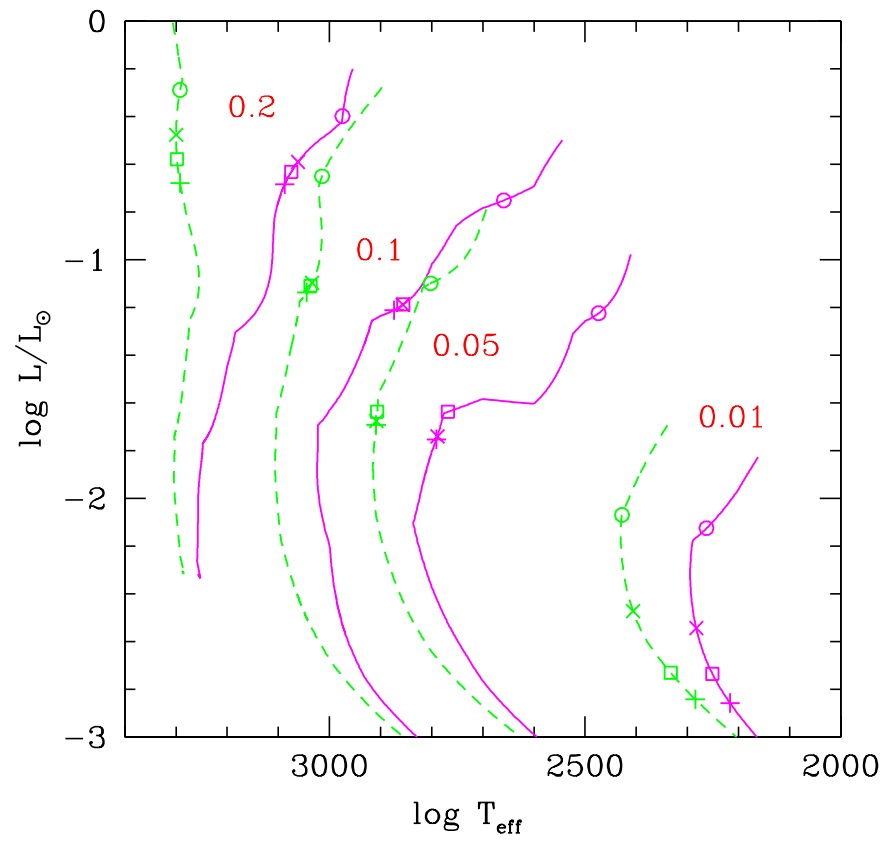

Fig. 6. Evolutionary tracks in the Hertzsprung-Russell diagram for masses from $0.2 M_{\odot}$ to $0.01 M_{\odot}$, as indicated. The solid curves correspond to $\alpha_{\text {mix }}=1$ and the dashed curves to $\alpha_{\text {mix }}=2$. Ages of 0.1 and $1 \mathrm{Myr}$ are indicated by respectively crosses and plus for models (A) with initial gravity $\log g=$ 3.0-3.5 and by open circles and squares for models (B) with initial $\log g=2.5$. Note that for models (A) the initial position (age $=0$ ) is essentially the same as the position at $0.1 \mathrm{Myr}$ (cross) and can differ significantly from position of models (B) at $t=0$.

to be taken into account for a correct age estimate of very young clusters.

\subsection{Convection}

One of the major uncertainties affecting the evolution of stars with masses $m \gtrsim 0.6 M_{\odot}$ is due to the treatment of convection. These stars show relatively extended superadiabatic outer layers during PMS and MS evolution, of which description is extremely sensitive to the adopted treatment of convection, i.e., within the MLT formalism, to the adopted mixing length (at any gravity). The effect of a variation of $l_{\text {mix }}$ on evolutionary tracks for solar-type stars is well known and is illustrated e.g. in Fig. $2 b$ of Baraffe et al. (2001). Ludwig et al. (1999) calibrated the mixing length parameter $\alpha_{\text {mix }}$ for these stars with 2D hydrodynamical models performed in the parameter space $4300 \mathrm{~K} \leq T_{\text {eff }} \leq 7100 \mathrm{~K}$ and gravities $2.54 \leq \log g \leq 4.74$. They found a moderate variation of the mixing length parameter around typically $\alpha=1.5$. Note that for the present models and input physics ${ }^{2}, l_{\text {mix }}=1.9 H_{\mathrm{P}}$ is the value required to fit the Sun at its present age. An increase of $\alpha_{\text {mix }}$

\footnotetext{
2 As stressed in BCAH98, the value of $l_{\text {mix }}$ required to fit the Sun depends on the input physics (equation of state, opacities, outer boundary condition).
}

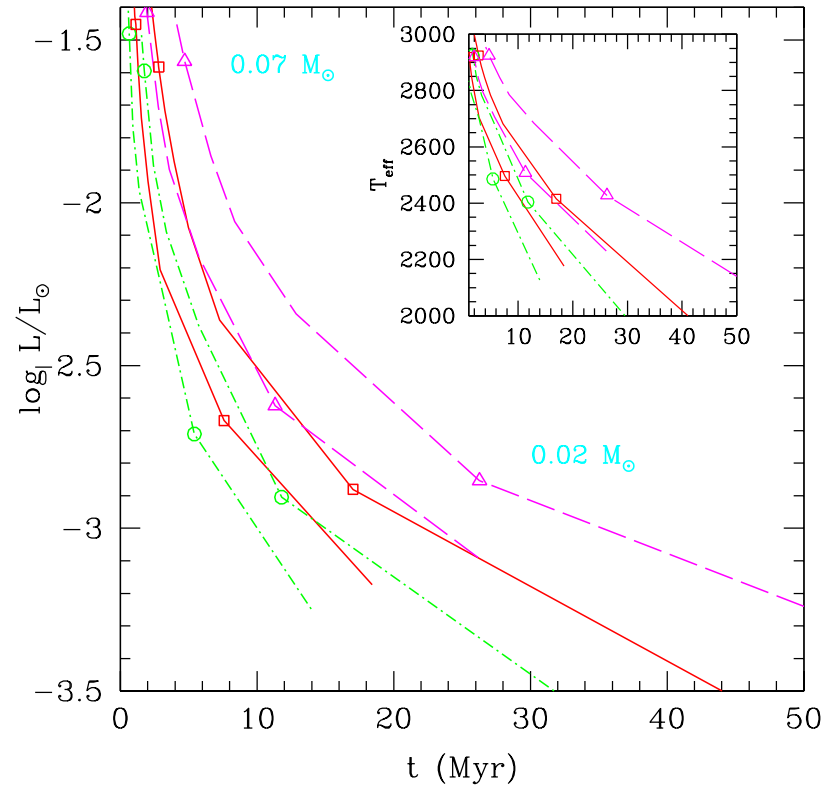

Fig. 7. Deuterium depletion curves as a function of age in $L$ and $T_{\text {eff }}$ for different initial $D$ abundances: $\left[{ }^{2} D_{0}\right]=10^{-5}$ (dashdotted curves), $\left[{ }^{2} D_{0}\right]=2 \times 10^{-5}$ (solid curves) and $\left[{ }^{2} D_{0}\right]=$ $4 \times 10^{-5}$ (dashed curve). For each initial value of $\left[{ }^{2} D_{0}\right]$, two curves are shown corresponding to the $50 \%$ (left curve) and $99 \%$ (right curve) depletion limits. The symbols on the curves indicate the location of respectively $0.07 M_{\odot}$ and $0.02 M_{\odot}$ brown dwarfs.

from 1 to 2 yields an increase of $T_{\text {eff }}$ up to $500 \mathrm{~K}$ for the highest masses during their PMS evolution.

For masses $m \lesssim 0.6 M_{\odot}$, the extension of the superadiabatic layers retracts appreciably and the transition from convective to radiative outer layers is characterized by an abrupt transition from a fully adiabatic to a radiative structure with a very small entropy jump. This means that during most of the evolution, except at early ages, as discussed in Sect. 3.1.1 and below, the sensitivity of the evolutionary models to $l_{\text {mix }}$ is small. Multi-dimensional hydrodynamical simulations for conditions characteristic of M-dwarf atmospheres, $T_{\text {eff }} \leq 3000 \mathrm{~K}, \log g=5$, have been recently conducted by Ludwig et al. (2001). These simulations confirm the afore-mentioned small entropy jump found in the 1-D models described by the MLT, illustrating the large efficiency of atmospheric convection for these objects, a direct consequence of the formation of molecules, as mentioned earlier. Under these circumstances, the 3D simulations show that the MLT does indeed provide a correct thermal profile, providing a value of $\alpha_{\text {mix }}>1$, at least for high gravities and relatively old objects $(t \gg 10$ Myr).

In Sect. 3.1.1, however, we have shown that even below $0.6 M_{\odot}$, very young models with gravities $\log g \lesssim$ 4 can be affected by a variation of $l_{\text {mix }}$. To minimize such uncertainties, a correct calibration of $l_{\text {mix }}$ requires the extension of the Ludwig et al. (2001) calculations to lower gravities. This work is under progress and represents the most promising method for an accurate description 
of convection in optically-thin media, through an accurate calibration of $l_{\text {mix }}$. In contrast, given all the already mentioned uncertainties inherent to either observation or models for very young objects, a calibration of $l_{\text {mix }}$ based on a comparison of PMS tracks with observations is at best highly speculative.

\section{Comparison with other work}

A detailed comparison between various evolutionary tracks available in the literature has already been done by Siess et al. (2000). In the present section, we compare the evolutionary tracks most widely used by the community to describe the observational properties of objects in young clusters (Burrows et al. 1997, B97; D'Antona \& Mazzitelli 1994, 1997, DM94, DM97; Palla \& Stahler 1999, PS99). The comparison between these different models in a theoretical HR diagram is illustrated in Fig. 8 for a few low-mass star and BD masses.

Let us first summarize the main differences between these models. One crucial difference is due to the outer boundary condition: DM94, DM97 and PS99 use approximate boundary conditions based on $T(\tau)$ relationships assuming gray approximation and radiative equilibrium. Outer boundary conditions based on such approximations are wrong as soon as molecules form near the photosphere and convection reaches optically thin layers, i.e. below $T_{\text {eff }} \sim 4000 \mathrm{~K}$ (see Chabrier \& Baraffe 1997, 2000; and references therein) They usually yield hotter effective temperatures for a given mass. Above $\sim 4000 \mathrm{~K}$, the choice of the outer boundary condition is less consequential, as shown by the good agreement between the $0.8 M_{\odot}$ tracks of BCAH98 and of PS99 (see Fig. 8), all the other physical inputs being similar for such mass. Note that, although the PS99 models start from a more realistic birthline, this does not affect the results after $1 \mathrm{Myr}$, as expected from the tests on initial conditions performed in Sect. 3.1.1.

The B97 models for low mass stars and hot brown dwarfs ( $T_{\text {eff }} \gtrsim 2000 \mathrm{~K}$ ) are based on gray atmosphere models obtained by solving the radiative transfer equation, as described in Burrows et al. (1993). Such an approximation, although it represents an improvement over the previous $T(\tau)$ relationships, still overestimates $T_{\text {eff }}$ at a given mass compared to models based on full non-gray atmosphere models. This is certainly the reason why for VLMS on the Main Sequence (for $m>0.08 M_{\odot}$ ), the B97 models are about 100-200 K hotter than the BCAH98 ones. We note, however, that the very early evolution of the B97 models proceeds at much hotter $T_{\text {eff }}$ than any other model. Although also true for masses below $0.2 M_{\odot}$, this does not appear in Fig. 8 since the tracks start at $1 \mathrm{Myr}$ and the $0.06 M_{\odot}$ of B97 displayed in Fig. 8 is indeed much hotter but for $t<1 \mathrm{Myr}$. The reasons for such differences along the Hayashi line is not clear. We only notice that similar shape of the Hayashi line (i.e. a strong decrease of $\left.T_{\text {eff }}\right)$ is obtained if one assumes a fully adiabatic initial structure and adiabatic convection (i.e. $\alpha_{\text {mix }} \rightarrow \infty$ ). Based on test cases, we find that such initial models are much hotter for a given $L$. If $T_{\text {eff }}$ is high enough for $\mathrm{H}_{2}$ formation to be negligible, such models start to evolve toward cooler $T_{\text {eff }}$. Once the fraction of $\mathrm{H}_{2}$ becomes significant and the adiabatic gradient small enough near the photosphere, evolution proceeds almost vertically in the HRD, as also expected from the analysis of Hayashi \& Nakano (1963) (see Sect. 3.1.1). Such an evolution resembles the shape of the B97 tracks. It may however be a pure coincidence, since B97 do not describe their initial conditions.

Convection in all afore-mentioned models is treated within the framework of the mixing length theory, with $1<\alpha_{\text {mix }}<2$, except DM97, who use the CanutoMazzitelli formalism. As demonstrated in Sect. 3, the treatment of convection affects significantly evolutionary tracks and a variation of $\alpha_{\text {mix }}$ modifies the shape of the Hayashi lines (as long as super-adiabatic layers are present). Figure 8 displays quite similar shapes for a given mass between BCAH98, PS99 and DM94, although not at the same $T_{\text {eff }}$ because of different outer boundary conditions. The DM97 Hayashi lines behave differently with respect to models of other groups, as already noticed by Siess et al. (2000). This is certainly due to their different treatment of convection. Unexpectedly, the DM97 models predict a MS for VLMS close to the BCAH98 MS, which, as mentioned by DM97, is purely coincidental and stems from unexpected canceling effects between different treatments of convection and outer boundary conditions. This is illustrated for the $0.2 M_{\odot}$ in Fig. 8. Since the grey-like outer boundary condition used in DM97 is expected to yield hotter effective temperatures than the BCAH98 models, the close agreement on the MS suggests that the Canuto-Mazzitelli convection treatment yields larger super-adiabatic layers in the atmosphere. This is indeed required to decrease $T_{\text {eff }}$ for a given mass. Such a behaviour, however, is not found by the recent simulations by Ludwig et al. (2001) (see Sect. 3.2). Indeed, the Canuto-Mazzitelli treatment of convection yields results at odds with 3D hydro simulations for the outer thermal profile of the Sun (see e.g. Nordlund \& Stein 1999), and does not provide an accurate treatment of convection in optically-thin media, at least for solar-type stars and lowmass stars ( see e.g. Sect. 2.1.3 of Chabrier \& Baraffe 2000 for a discussion of this topic).

\section{Observational tests}

In the previous sections, we emphasize the large uncertainty of evolutionary models at early ages and low gravities. Testing different initial conditions, we find out that after a few Myr these uncertainties become inconsequential and all our models converge toward the same position in a HRD at a given age, for a given mass. Such a result does not necessarily imply that the models are reliable at ages of a few Myr, since we have only tested simple cases. More sophisticated initial conditions are beyond the scope of the present paper and were already explored by Hartman et al. (1997), illustrating the sensitivity of tracks and birthline positions to the (poorly known) details of 


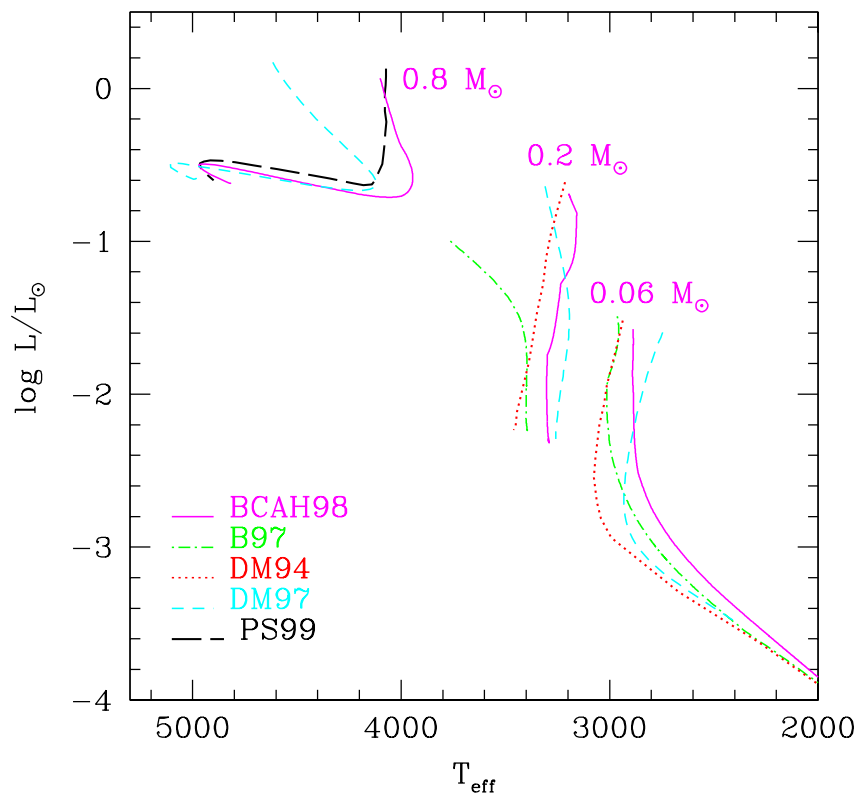

Fig. 8. Comparison of evolutionary tracks from different authors, as indicated on the figure. All models start at $t=1 \mathrm{Myr}$, and evolve up to the MS for the 0.8 and $0.2 M_{\odot}$ stars.

the protostellar accretion process (geometry, rate, temperature of added matter).

A better knowledge of initial conditions may come from the determination of the minimum age below which present models start to depart significantly from observations. Estimation of this age can constrain the characteristic time-scales and accretion rates of the protostellar collapse phase. Unfortunately, direct comparisons of observations with models directly in colour-magnitude diagrams are extremely uncertain due to the large extinction in star formation regions, which affects the observed energy distribution and thus the spectra and the colors. Only very few exceptions, such as $\sigma$ Orionis, exhibit low extinction. Recently, Béjar et al. (1999) and Zapatero et al. (1999, 2000) obtained optical and near-IR photometry for low mass objects in this cluster. In a $(I-J)$ vs. $M_{\text {I }}$ CMD, the data lie between the 1 and 10 Myr isochrones, respectively, for masses down to $\sim 0.01 M_{\odot}$, using the BCAH98 and CBAH00 models (Zapatero et al. 2000; Béjar et al. 2001). If statistics is improved and if the membership of the objects to the cluster is confirmed, such observations provide an unique opportunity to test directly the validity of young theoretical isochrones. They also offer the best chance to determine the Initial Mass Function (IMF) down to the substellar regime and the minimum mass formed by a collapse process (see Béjar et al. 2001).

Young multiple systems provide also excellent tests for PMS models at young ages, because of the assumed coevality of their different components. In addition, another strong constraint is supplied by the estimate of dynamical masses deduced either from binary systems (Covino et al. 2000; Steffen et al. 2001) or determined from the orbital motion of circumstellar/circumbinary disks (Simon et al. 2000). An example is provided by the quadruple system
GG TAU (White et al. 1999), with components covering the whole mass-range of VLMS and BDs from $1 M_{\odot}$ to $\sim 0.02 M_{\odot}$. Orbital velocity measurements of the circumbinary disk surrounding the two most massive components imply a constraint on their combined stellar mass (Dutrey et al. 1994; Guilloteau et al. 1999). This mass constraint and the hypothesis of coevality provides a stringent test for PMS models. The BCAH98 models are the most consistent with GG Tau (for details see White et al. 1999; Luhman 1999) and provide the closest agreement with derived masses of other young systems (see Fig. 9).

Most of the observed systems displayed in Fig. 9 are better reproduced by tracks using a large value of $\alpha_{\text {mix }}$ (=1.9). However, for some systems, such as 1 (Covino et al. 2000), 2 (Steffen et al. 2001) and 4 (BP Tau from Simon et al. 2000), a better agreement is obtained with $\alpha_{\text {mix }}=$ 1. Although a variation of $\alpha_{\text {mix }}$ with effective temperature and gravity is possible, as suggested by the simulations of Ludwig et al. (1999), none of these three systems occupies a peculiar position in $\left(T_{\text {eff }}, g\right)$ to suggest a different value of $\alpha_{\text {mix }}$. This puzzle may reflect the uncertainties of PMS models based on arbitrary initial conditions. It may also be due to the large uncertainties of observationally-derived spectral type classifications, luminosity estimates and $T_{\text {eff }}$ calibrations for such very young objects. Since they display spectral features between that of giants and dwarfs (see Luhman 1999), a better representation of their spectral properties may require new indices more appropriate to these intermediate surface gravities, in the same vein as the pseudo-continuum ratios used by Martín et al. (1996) for Pleiades objects. The transformation of the inferred spectral type into $T_{\text {eff }}$ is even more difficult, because of the lack of reliable $T_{\text {eff }}$-scales for such young T-Tauri like objects. Significant efforts were devoted within the past recent years to the elaboration of improved $T_{\text {eff-scales for M-dwarfs (Leggett et al. 1996) }}$ and M-giants (Perrin et al. 1998; van Belle et al. 1999). However, work remains to be done for T-Tauri like objects. Luhman (1999) defined a $T_{\text {eff-scale intermediate }}$ between giants and dwarfs and based on the isochrone of BCAH98 which goes through the four components of GG Tau. Interestingly enough, applying this $T_{\text {eff-scale to }}$ young clusters such as IC 348 (Luhman 1999) and star forming regions like Chamaeleon I (Comerón et al. 2000), the cluster members show a small scatter in age and no obvious correlation between age and mass. As mentioned by Comerón et al. (2000), this suggests in Chamaeleon I an almost coeval population which formed within less than 1 Myr. This short timescale supports the suggestion that star formation is controlled primarily by large-scale turbulent flows rather than by magnetic processes such as ambipolar diffusion (Hartmann 2000).

Given the assumption of coevality and the uncertainties of our initial conditions, the $T_{\text {eff }}$-scale for young objects suggested by Luhman (1999) needs to be confirmed in order to confirm these exciting results about star formation process. Although still very preliminary, the comparison of observed and synthetic spectra, as recently done by 


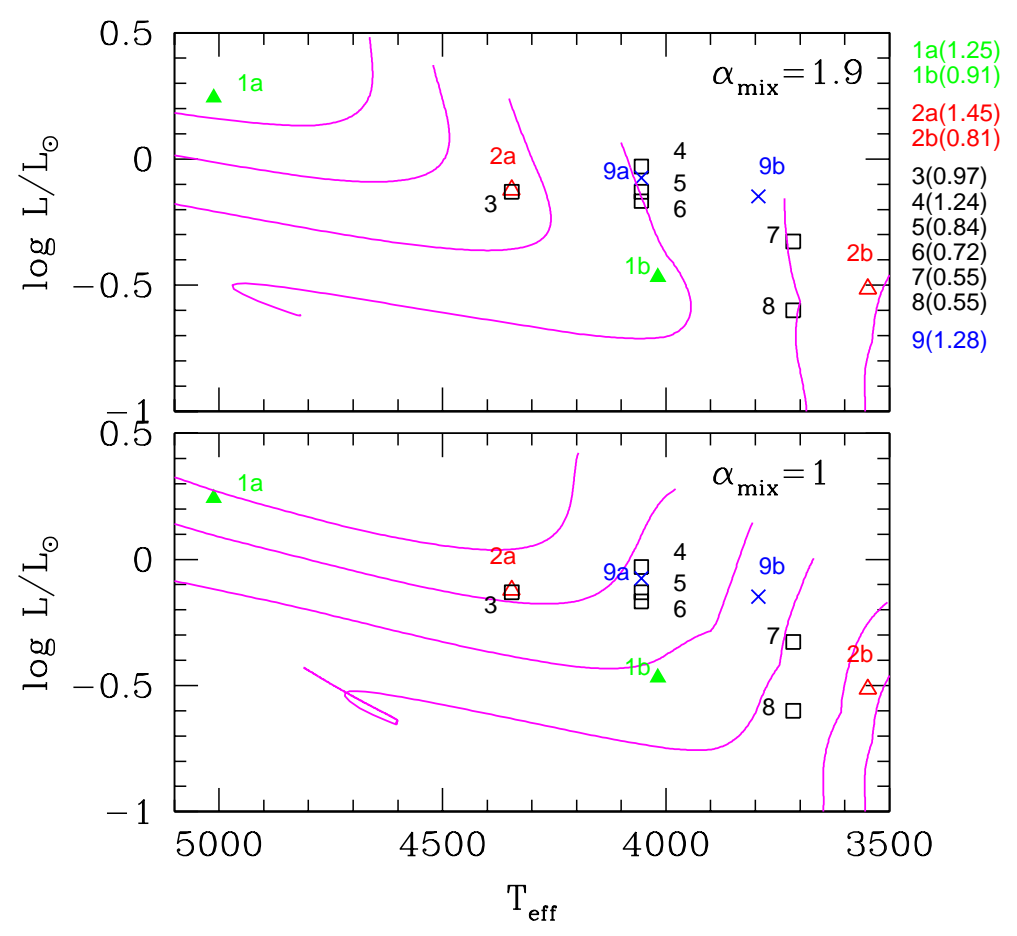

Fig. 9. Comparison of evolutionary tracks with observed PMS objects with derived masses. The BCAH98 tracks are displayed for 1.4, 1.2, 1, 0.8, 0.6 and $0.5 M_{\odot}$ (from left to right) for two values of $\alpha_{\text {mix }}$. Observations are from Covino et al. (2000, filled triangles), Simon et al. (2000, crosses and open squares) and Steffen et al. (2001, open triangles). Open squares are single objects whereas all the other symbols indicate binaries. For each systems, masses are indicated (in $M_{\odot}$ ) on the right hand side of the figure. For GG tau A $(9 \mathrm{a}+9 \mathrm{~b}$, crosses $)$ the total mass is indicated.

Lucas et al. (2001) for Orion objects, provides a promising way to define such a $T_{\text {eff- }}$-scale.

\section{Conclusion}

The very good agreement of models based on improved physics with observations for relatively old ( $t \gtrsim 100 \mathrm{Myr}$ ) low-mass objects yields confidence in the underlying theory. Such evolutionary models can now be confronted to the complex realm of very young objects, providing important information on star formation processes and initial conditions for PMS models. Although based on extremely simple initial conditions (no accretion phase, no account of protostellar collapse phase and time scale, spherical symmetry), these models provide the most accurate comparison with present observations of very young objects (dynamical masses, tests of coevality in multiple systems, CMDs). Given the combining effects of large observational and theoretical uncertainties at very young ages, however, one must remain cautious. It is probably too premature to conclude on the validity of the present models at early phases of evolution. We have examined in the present paper the uncertainties on evolutionary models of very-young low-mass objects arising from initial conditions, in particular the initial radius of the object, the efficiency of convection in the outermost layers and the initial abundance of deuterium. We have shown that at least the two first afore-mentioned uncertainties can affect drastically the fundamental properties, luminosity and effective temperature of objects younger than about 1 Myr. Therefore, any attempt to infer an age or a mass from observable quantities for these objects, in particular the initial mass function of very young clusters, must be considered with highly limited - if any - validity!

Realistic initial conditions can only be provided by multi-dimensional protostar collapse simulations, not by spherically-symmetric models for PMS initial conditions involving many free, ill- or unconstrained parameters. Because of numerical subtleties and complex physical processes (accretion fronts, turbulent time-dependent convection, hydrodynamical radiative transfer, magnetic field etc.), the construction of star formation models is a harsh task, which very likely will necessitate several years of efforts. Besides these theoretical difficulties, observations of very young objects can provide only limited guidance to such simulations, since most phases involved during the collapse are embedded in dusty cocoons. Only the final product can be observationally tested, when the protostar becomes visible. This stage marks essentially the beginning of PMS evolutionary tracks. PMS tracks tested against observations thus provide a precious link to gather insight about star formation models from subsequent evolution.

To progress in the field and in parallel with the development of star formation models, efforts can be directed toward: (1) a reduction of the main theoretical uncertainties affecting PMS models at low gravities, which involves in particular a better determination of $l_{\text {mix }}$ through 
multi-D hydrodynamical simulations and (2) the elaboration of a reliable $T_{\text {eff-scale for young objects. }}$

Note: Tracks and isochrones for $t \geq 1 \mathrm{Myr}$ of the BCAH98 models (from $0.02 M_{\odot}$ to $1.4 M_{\odot}$ ) and of the CBAH00 models including dusty atmospheres (from $0.001 M_{\odot}$ to $0.1 M_{\odot}$ ) are available by anonymous ftp:

ftp ftp.ens-lyon.fr

username: anonymous

ftp $>$ cd /pub/users/CRAL/ibaraffe

ftp $>$ get README

ftp $>$ get BCAH98_models.*

ftp $>$ get BCAH98_iso.*

ftp $>$ get DUSTY00_models

$\mathrm{ftp}>$ quit

Acknowledgements. We are indebted to Lee Hartmann for valuable discussions. I.B. thanks the Max-Planck-Institut for Astrophysik in Garching for hospitality during elaboration of part of this work. The calculations were performed using facilities at Centre d'Études Nucléaires de Grenoble.

\section{References}

Allard, F., Hauschildt, P. H., Alexander, D. R., \& Starrfield, S. 1997, ARA\&A, 35, 137

Allard, F., Hauschildt, P. H., \& Schweitzer, A. 2000, ApJ, 539, 366

Allard, F., Hauschildt, P. H., Alexander, D. R., Tamanai, A., \& Schweitzer, A. 2001, ApJ, 556, 357

Baraffe, I., Chabrier, G., Allard, F., \& Hauschildt, P. H. 1995, ApJ, 446, L35

Baraffe, I., Chabrier, G., Allard, F., \& Hauschildt, P. H. 1997, A\&A, 327, 1054

Baraffe, I., Chabrier, G., Allard, F., \& Hauschildt, P. H. 1998, A\&A, 337, 403 (BCAH98)

Baraffe, I., Chabrier, G., Allard, F., \& Hauschildt, P. H. 2001, From darkness to light: origin and evolution of young stellar clusters, ASP Conf. Ser. 243 (Cargese 2000) [astro-ph/0007157]

Béjar, V. J. S., Zapatero Osorio, M. R., \& Rebolo, R. 1999, ApJ, 521, 671

Béjar, V. J. S., Martín, E. L., Zapatero Osorio, M. R., et al. 2001, ApJ, 556, 830

Burrows, A., \& Liebert, J. 1993, Rev. Mod. Phys., 65, 301

Burrows, A., Hubbard, W. B., Saumon, D., \& Lunine, J. I. 1993, ApJ, 406, 158

Burrows, A., Marley, M., Hubbard, W. B., et al. 1997, ApJ, 491, 856 (B97)

Chabrier, G., \& Baraffe, I. 1997, A\&A, 327, 1039

Chabrier, G., \& Baraffe, I. 2000, ARA\&A, 38, 337

Chabrier, G., Baraffe, I., Allard, F., \& Hauschildt, P. H. 2000a, ApJ, 542, 464 (CBAH00)

Chabrier, G., Baraffe, I., Allard, F., \& Hauschildt, P. H. 2000b, ApJ, 542, 119

Comerón, F., Neuhäuser, R., \& Kaas, A. A. 2000, A\&A, 359, 269
Covino, E., Catalano, S., Frasca, A., et al. 2000, A\&A, 361, L49

D'Antona, F., \& Mazzitelli, I. 1994, ApJS, 90, 467 (DM94)

D'Antona, F., \& Mazzitelli, I. 1997, in Cool stars in Clusters and Associations, ed. R. Pallavicini, \& G. Micela, Mem. S. A. It., 68, 807 (DM97)

Dutrey, A., Guilloteau, S., \& Simon, M. 1994, A\&A, 286, 149

Guilloteau, S., Dutrey, A., \& Simon, M. 1999, A\&A, 348, 570

Hartmann, L. 2000, in Proc. of 33rd ESLAB Symp., Star formation from the small to the large scale, ed. F. Favata, A. A. Kaas, \& A. Wilson, ESA SP-445

Hartmann, L., Cassen, P., \& Kenyon, S. J. 1997, ApJ, 475, 770

Hauschildt, P. H., Allard, F., \& Baron, E. 1999a, ApJ, 512, 377

Hauschildt, P. H., Allard, F., Ferguson, J., Baron, E., \& Alexander, D. R. 1999b, ApJ, 525, 871

Hayashi, C. 1961, PASJ, 13, 450

Hayashi, C., \& Nakano, T. 1963, Prog. Theor. Phys., 30, 460

Leggett, S. K., Allard, F., Berriman, G., Dahn, C. C., \& Hauschildt, P. H. 1996, ApJS, 104, 117

Linsky, J. 1998, Space Sci. Rev., 84, 285

Lucas, P. W., Roche, P. F., Allard, F., \& Hauschildt, P. H. 2001, MNRAS, 326, 695

Ludwig, H. G., Freytag, B., \& Steffen, M. 1999, A\&A, 346, 111

Ludwig, H. G., Allard, F., \& Hauschildt, P. H. 2001, A\&A, submitted

Luhman, K. L. 1999, ApJ, 525, 466

Martín, E. L., Rebolo, R., \& Zapatero Osorio, M. R. 1996, ApJ, 469, 706

Miller, S., Tennyson, J., Jones, H. R. A, \& Longmor, A. J. 1994, in Molecules in the Stellar Environment, ed. U. G. Jorgensen, Lecture Notes in Physics

Nordlund, A., \& Stein, R. F. 1999, Theory and Tests of Convection in Stellar Structure, ASP Conf. Ser., 173, 91

O'Meara, J. M., Tytler, D., Kirkman, D., et al. 2001, AAS, 197,5604

Palla, F., \& Stahler, S. W. 1999, ApJ, 525, 772 (PS99)

Partridge, H., \& Schwenke, D. W. 1997, J. Chem. Phys., 106, 4618

Perrin, G., Coudé du Foresto, V., Rigway, S. T., et al. 1998, A\&A, 331, 619

Saumon, D., Hubbard, W. B., Burrows, A., et al. 1996, ApJ, 460, 993

Schwenke, D. W. 1998, Chemistry and Physics of Molecules and Grains in Space, Faraday Discussion, 109, 321

Siess, L., Dufour, E., \& Forestini, M. 2000, A\&A, 358, 593

Simon, M., Dutrey, A., \& Guilloteau, S. 2000, ApJ, 545, 1034

Stahler, S. W. 1983, ApJ, 274, 822

Stahler, S. W. 1988, ApJ, 332, 804

Stahler, S. W., Shu, F. H., \& Taam, R. E. 1980, ApJ, 241, 637

Steffen, A. T., Mathieu, R. D., Lattanzi, M. G., et al. 2001, AJ, 122, 997

van Belle, G. T., Lare, B. F., Thompson, R. R., et al. 1999, AJ, 117, 521

White, R. J., Ghez, A. M., Reid, I. N., \& Schultz, G. 1999, ApJ, 520, 811

Zapatero Osorio, M. R., Béjar, V. J. S., Rebolo, R., Martín, E. L., \& Basri, G. 1999, ApJ, 524, L115

Zapatero Osorio, M. R., Béjar, V. J. S., Martín, E. L., et al. 2000, Science, 290, 103 